\title{
Entwicklung der Sozialen Dienste der Strafjustiz aus dem Blickwinkel der amtlichen Statistiken
}

Peggy Schäpler

$\mathrm{B}$ ewährungshilfe, Gerichtshilfe und Führungsaufsicht nehmen vor dem Hintergrund der Verhinderung weiterer Straftaten durch Resozialisierung und Kontrolle unverzichtbare Aufgaben innerhalb einer sozialen Strafrechtspflege wahr und setzen dabei zugleich Regelungen und Konzepte um, die der Haft- und damit Kostenvermeidung dienen. Während in den alten Bundesländern im Hinblick auf Personalfragen und Fallbearbeitung zumeist von einer Trennung zwischen den drei Bereichen ausgegangen wird, erfolgte in Mecklenburg-Vorpommern, Brandenburg und Sachsen-Anhalt im Zuge des Aufbaus der sozialen Strafrechtspflege durch eine Personalunion die Schaffung eines einheitlichen Sozialen Dienstes der Justiz. Sachsen vollzog den Zusammenschluss erst im Jahr 1996, allerdings ohne ausdrückliche Regelung der durchgehenden Betreuung1. Wenn es nun um die Entwicklung der Sozialen Dienste geht, so wirft dies auch immer die Frage nach Unterstellungszahlen, Erfolgsquoten, Probandenbelastung etc. auf. Die dahingehend vorhandenen offiziellen Datenquellen sind sehr begrenzt und vor allem nicht auf dem neusten Stand. So wurde erst im Jahr 2003 durch das Statistische Bundesamt die Bewährungshilfestatistik für die Jahre 1998/99 herausgegeben. Im Sicherheitsbericht der Bundesregierung aus dem Jahr 2001 musste im Bereich der Bewährungshilfe auf Zahlen von 1997 zurückgegriffen werden. Weiterhin wird eine Vergleichbarkeit der Daten der einzelnen Bundesländer aufgrund unterschiedlicher Erhebungsmethoden bezweifelt. Jedoch ist in Planung die Bewährungshilfestatistik des Statistischen Bundesamtes ab dem Jahr 2004 wieder kontinuierlich her- auszugeben. Dafür wurden im Vorfeld die Erhebungsinstrumente abgeglichen, um Fehlerquellen $\mathrm{zu}$ beseitigen und damit eine bessere Vergleichbarkeit zu ermöglichen.

Der nun folgende Beitrag zur Entwicklung der Sozialen Dienste bezieht sich auf die bundesweiten Daten bis 1999 bzw. verwendet Informationen die dankenswerterweise durch das Justizministerium Mecklenburg-Vorpommern zur Verfügung gestellt wurden. Weitergehende Umfragen sind von Seiten des Lehrstuhls für Kriminologie in Greifswald geplant.

\section{Bewährungshilfe}

Die Arbeit der Bewährungshilfe umfasst neben der Betreuung auch die Kontrolle der Unterstellten u.a. im Hinblick auf die Erfüllung der vom Gericht erteilten Auflagen und Weisungen (vgl.
§ 56d III StGB). Im Fall der Strafaussetzung zur Bewährung ist für Jugendliche gemäß § 24 JGG die Unterstellung unter die Aufsicht der Bewährungshilfe obligatorisch, für Erwachsene prognoseabhängig nach § 56d StGB. Seit ihrer Einführung im Jahr 1953 zeichnet sich die Arbeit der Bewährungshilfe durch steigende Akzeptanz auf Seiten der Richter- und Staatsanwaltschaft aus. Ein Blick in die Unterstellungszahlen bestätigt diese Annahme. So ist für das frühere Bundesgebiet ein Anstieg der Zahl der Unterstellungen unter Bewährungsaufsicht insgesamt im Zeitraum 1963-1999 stichtagsbezogen (31.12.) von absolut 27.401 auf $148.633 \mathrm{zu}$ verzeichnen, der Ausgangswert hat sich damit mehr als verfünffacht.

Im folgenden Schaubild wurden die Unterstellungen insgesamt in Unterstellungen nach allgemeinem Strafrecht bzw. nach Jugendstrafrecht für den genannten Zeitraum aufgeschlüsselt. Im Bereich der Unterstellungen nach allgemeinem

Schaubild: Entwicklung der Unterstellungen unter Bewährungsaufsicht nach allgemeinem Strafrecht und nach Jugendstrafrecht, früheres Bundesgebiet 1963-1999*

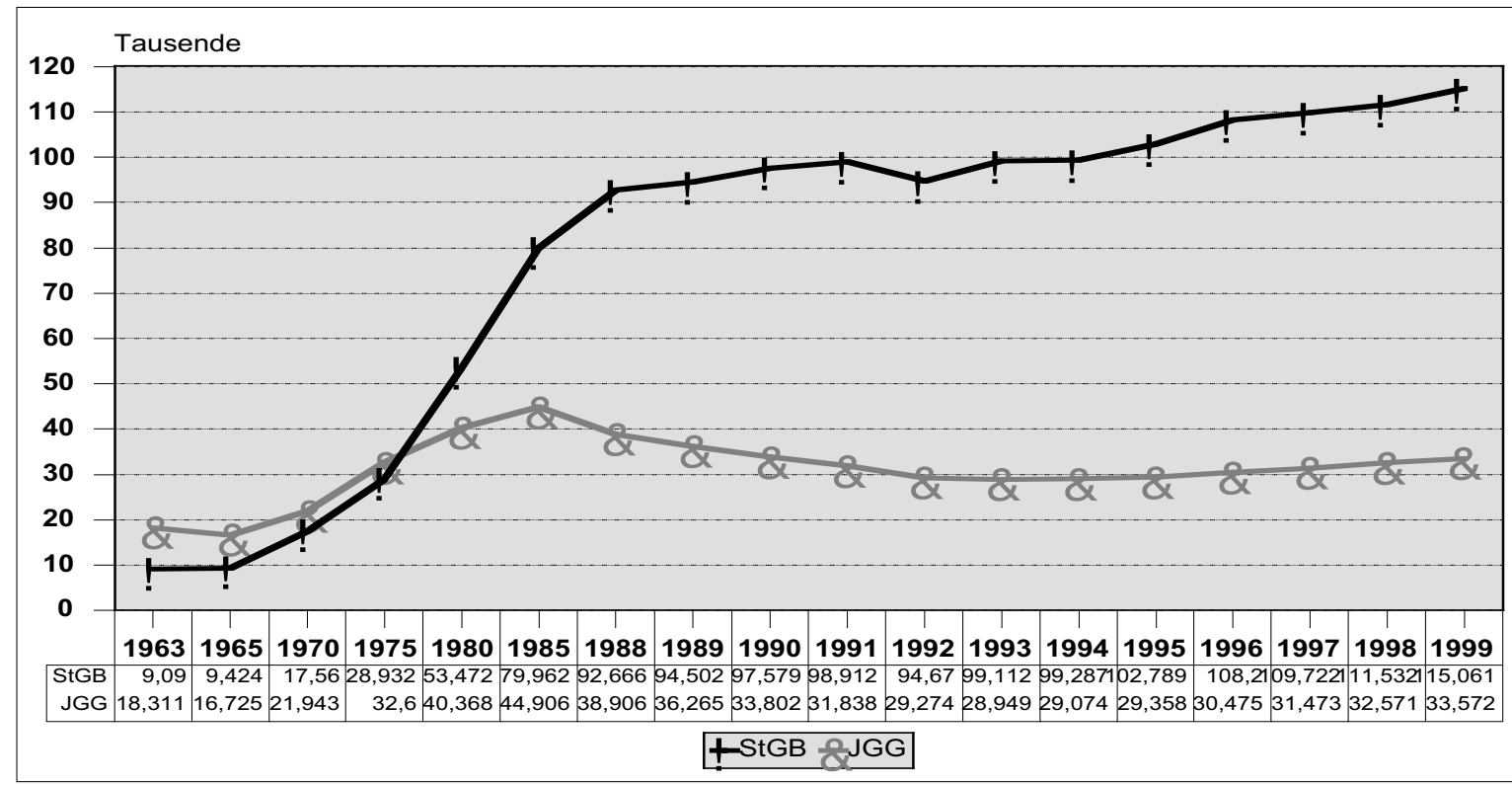

*seit 1992 einschließlich Gesamt-Berlin, aber ohne Hamburg

Quelle: Statistisches Bundesamt, Bewährungshilfestatistik 1998/1999, Fachserie 10, Reihe 5 
Strafrecht ist ein dynamischer Anstieg der Unterstellungszahlen zu sehen. Die Unterstellungen nach Jugendstrafrecht erreichten 1985 einen Höchstwert, der weitere Verlauf könnte nach einer Abnahme seit 1992 eher als stagnierend bezeichnet werden. Der Rückgang seit 1985 bzw. der stabile Verlauf seit Anfang der 90iger Jahre dürfte zwei Ursachen haben: zum einen der Bedeutungszuwachs anderer ambulanter Sanktionen wie Betreuungsweisungen, soziale Trainingskurse oder Täter-Opfer-Ausgleich ${ }^{2}$, zum anderen der demographische Faktor sinkender Geburtenraten. Der enorme Zuwachs der Unterstellungszahlen in den letzten Jahren ist damit auf den Anstieg der Unterstellungen nach allgemeinem Strafrecht zurückzuführen, der sich besonders nach der Strafrechtsreform Mitte der siebziger Jahre bemerkbar machte ${ }^{3}$.

In der folgenden Tabelle 1 wurden, bezogen auf die letzte Stichtagserhebung (31.12.1999), die Unterstellungszahlen nach dem Grund der Unterstellungen erfasst. Wie bereits im ersten periodischen Sicherheitsbericht in einer entsprechenden Tabelle für 1997 ersichtlich, überwogen auch im Jahr 1999 sowohl bei den Unterstellungen nach allgemeinem Strafrecht als auch nach Jugendstrafrecht die Unterstellungen aufgrund primärer Strafaussetzung, die Quote lag jeweils zwischen $60 \%$ bzw. 70\%. Zudem waren bei der Reststrafenaussetzung mehrheitlich Fälle betroffen, in denen die Reststrafe unter einem Jahr blieb.

Eine Differenzierung der Bewährungsunterstellungen aus dem Jahr 1999 nach der Deliktsstruktur zeigt, dass Eigentums- und Vermögensdelikte mit zusammen rund $42 \%$ die größte Gruppe ausmachten. Fasst man die Delikte gegen die Person, d. h. Körperverletzungs- und Tötungsdelikte und die Raubdelikte unter dem Begriff der Gewaltkriminalität zusammen, so handelt es sich hierbei mit 20\% um die zweitstärkste Gruppe, gefolgt von Straftaten gegen das Betäubungsmittelgesetz mit 16\% und Straftaten im Straßenverkehr mit $10 \%{ }^{4}$. Insgesamt deutet sich an, dass die Probanden der Bewährungshilfe schon von der Deliktsstruktur her gesehen keine Bagatellfälle, sondern in erheblichem Umfang strafrechtlich vorbelastete Fälle mit Problemen sozialer Desintegration betreffen, wie sie auch aus den früheren Studien zur Bewährungshilfe bekannt sind ${ }^{5}$. Probanden der
Bewährungshilfe weisen damit weitgehende Überschneidungen mit der Strafvollzugspopulation auf.

Angesichts der hohen Unterstellungszahlen und der schwierigen Klientel sind natürlich die Bewährungs- bzw. Widerrufsquoten von großem Interesse. Die Statistiken deuten insoweit auf einen herausragenden Erfolg der Institution Bewährungshilfe hin. 1999 lag die durchschnittliche Widerrufsquote bei beendeten Unterstellungen nach allgemeinem Strafrecht für das frühere Bundesgebiet bei rund 32\%, d.h. es wurden $68 \%$ der Beendigungen durch Bewährung abgeschlossen. Noch günstiger stellte sich die Situation dar, wenn es um beendete Unterstellungen nach dem Jugendstrafrecht geht. Nimmt man die beendeten Unterstellungen durch Einbeziehung in ein neues Urteil heraus, denn es handelt sich hier im Grunde um Rückfalltaten, die allerdings nicht zu einem Widerruf, sondern zu erneuter Unterstellung führten und insoweit zumindest kein Scheitern der Bewährungshilfe darstellen, lag die Bewährungsquote bundesweit durchschnittlich bei rund $75 \%$. Die positive Bilanz erscheint umso erstaunlicher, wenn man die durchschnittliche Probandenbelastung der einzelnen Bewährungshelfer erfasst. Tabelle 2 beruht auf einer Zusammenstellung des Hessischen Justizministeriums für das Jahr 2000 und zeigt je nach Bundesland die Anzahl der Unterstellungen, die Anzahl der Bewährungshelfer und die sich damit ergebenen durchschnittliche Belastung.
Danach lag die durchschnittliche Probandenbelastung im Jahr 2000 bundesweit für Bewährungshelfer bei ungefähr 70 Probanden. Ob man von einer einigermaßen angemessenen Betreuungssituation bei dieser Fallbelastung ausgehen kann, ist zweifelhaft. Darüber hinaus müssen die Zahlen für die Bundesländer mit einem einheitlichen Sozialen Dienst nach oben korrigiert werden, denn die Bewährungshelfer bearbeiten zusätzlich auch noch Gerichtshilfe- und Führungsaufsichtsfälle. So ergab die interne Statistik des Justizministeriums Mecklenburg-Vorpommern eine Gesamtbelastung von durchschnittlich 96 Probanden pro Bewährungshelfer/in im Jahr 2000 in M-V. Die Fallbelastung in einigen Regionen, wie im Landgerichtsbezirk Stralsund mit 116 Probanden pro Bewährungshelfer/in, war noch weit dramatischer als durch die Durchschnittszahlen des Landes bereits evident. Gespräche mit Bewährungshelfern dieses Landgerichtsbezirkes ergaben, dass sich die Situation gegenwärtig sogar noch verschlechtert hat: so muss man vereinzelt von einer Fallbelastung von bis zu 130 Probanden ausgehen.

Sicherlich ist die Situation nicht in allen Bundesländern so dramatisch, aber es stellt sich doch zunehmend die Frage, ob die Erfolgsquoten nicht auch auf mangelnder Kontrolle beruhen. Es erscheint offensichtlich, dass trotz der statistisch gesehen positiven Bilanz diese durchschnittlichen Fallzahlen bei Weitem die Leistungsfähigkeit der Bewährungshilfe als Teil der Sozialen Dienste der Justiz übersteigen. 


\section{Gerichtshilfe}

Das Institut der Gerichtshilfe wurde 1975 in die StPO eingeführt. Die Gerichtshilfe wird im Ermittlungsverfahren zur Aufklärung der sozialen und persönlichen Hintergründe der Tat eingeschaltet (vgl. § 160 III StPO) sowie im Vollstreckungsverfahren u.a. im Rahmen der Ersatzfreiheitsstrafenvermeidung (vgl. § 459d StPO). Allerdings gibt es keine offizielle Statistik anhand derer Aussagen über die Anzahl der Gerichtshelfer in der Bundesrepublik und deren Fallbelastung etc. getroffen werden könnten. Zudem unterscheidet sich die Organisationsstruktur zwischen den Bundesländern mit Personalunion und den Bundesländern mit einem eher traditionellen Verständnis. Aus diesen Gründen soll an dieser Stelle nur beispielhaft auf zwei Bundesländer eingegangen werden. In Mecklenburg-Vorpommern gibt es aufgrund der Personalunion keine klassischen Gerichtshelfer. Gerichtshilfe gehört wie auch die Bewährungshilfe und die Führungsaufsicht zum Sozialen Dienst der Justiz, der den Landgerichten zugeordnet ist. Im Jahr 2000 wurden in $\mathrm{M}-\mathrm{V}$ im Arbeitsfeld Gerichtshilfe 2.056 Aufträge bearbeitet, d.h. 27\% der Aufträge des Sozialen Dienstes in diesem Jahr waren Gerichtshilfefälle. Unter der Annahme von 56 zu dieser Zeit in der Fallarbeit tätigen Gerichts- und Bewährungshelfern ergab sich damit eine zusätzliche Belastung von rund 36 Gerichtshilfeaufträgen pro Mitarbeiter/in. Die Gerichtshilfe wird in M-V primär in der Ermittlungsarbeit tätig. Andere Aufgaben wie der Täter-OpferAusgleich oder die Vermittlung in gemeinnützige Arbeit wurden weitgehend an freie Träger übergeben.

Anders gestaltet sich die Situation in Niedersachsen. Dort sind insgesamt ca. 30 Gerichtshelfer in 11 Gerichtshilfestellen tätig. Die Gerichtshilfestellen sind den Staatsanwaltschaften angegliedert. Neben der Tätigkeit als Ermittlungsorgan und Haftentscheidungshilfe führen die Gerichtshelfer auch den Täter-Opfer-Ausgleich sowie die Vermittlung und Überwachung gemeinnütziger Arbeit im Rahmen des Programms »Schwitzen statt Sitzen « durch.

\section{Führungsaufsicht}

Die Führungsaufsicht wurde 1975 als Maßregel der Besserung und Sicherung eingeführt. Diese von Anfang an zum Teil sehr umstrittene Regelung hat eine Doppelfunktion: zum einen soll sie Tätern mit einer schlechten Sozialprognose Hilfen bei der Lebensführung gewähren, zum anderen diese Personen überwachen, um sie von der Begehung weiterer Straftaten abzuhalten (vgl. § 68a StGB) ${ }^{6}$. Wie bei der Gerichtshilfe ist es auch im Bereich der Führungsaufsicht schwierig Aussagen über die Anzahl der Unterstellungen, die Fallbelastung etc. zu treffen. Zum Teil werden die Führungsaufsichtsfälle in den Landesstatistiken, z.B. in Mecklenburg-Vorpommern, nicht getrennt ausgewiesen, sondern mit den Unterstellungen unter Bewährungshilfe vermischt. So soll auch hier beispielhaft auf zwei Bundesländer eingegangen werden. In Mecklenburg-Vorpommern bearbeiten die Mitarbeiter des Sozialen Dienstes der Justiz neben Gerichts- und Bewährungshilfe- auch Führungsaufsichtsfälle. Am Stichtag 31.12.2000 wurden 220 Aufträge im Arbeitsfeld Führungsaufsicht registriert. Im Hinblick auf das Gesamtauftragsvolumen des Sozialen Dienstes im Jahr 2000 nahmen Führungsaufsichtsfälle dementsprechend nur einen Anteil von 3\% ein.

In Niedersachsen sind die Führungsaufsichtsstellen den Landgerichten angegliedert. Insgesamt gibt es in Niedersachsen 11 Führungsaufsichtsstellen mit ca. 25 Mitarbeitern, die zumeist erfahrene Bewährungshelfer sind. Die Führungsaufsicht betreut und überwacht derzeit ca. 1.400
Probanden, das sind ca. 8\% aller unter der Aufsicht von Bewährungshelfern stehenden Probanden in Niedersachsen. Wie die Beispiele andeuten spielt die Führungsaufsicht innerhalb der Sozialen Dienste der Justiz eine eher untergeordnete Rolle ${ }^{7}$.

\section{Fazit}

Aussagen über die Entwicklung der Sozialen Dienste der Justiz mit Hilfe der amtlichen Statistiken zu treffen ist aufgrund der genannten Probleme sehr schwierig. Im Hinblick auf die vielfach geforderte Transparenz ist eine Verbesserung dieser Situation allerdings notwendig und ein erster Schritt in diese Richtung wird zweifellos die geplante Neuauflage der Bewährungshilfestatistik des Statistischen Bundesamtes darstellen.

\section{Literatur:}

Block, P. (1997): Rechtliche Strukturen der Sozialen Dienste in der Justiz, 2. Auflage, Wiesbaden: Kriminologische Zentralstelle.

Dünkel, F.; Geng, B., Kirstein, W. (1998): Soziale Trainingskurse und andere neue ambulante Maßnahmen nach dem JGG in Deutschland. Hrsg. vom Bundesministerium der Justiz - Mönchengladbach: Forum Verlag Godesberg.

Dünkel, F.; Spieß, G. (1992): Perspektiven der Strafaussetzung zur Bewährung und Bewährungshilfe im zukünftigen deutschen Strafrecht. In: BewHi 39, S. 117 - 138.

Tabelle 2: Probanden und Bewährungshelfer im Jahr 2000*

\begin{tabular}{|l|c|c|c|}
\hline Bundesland & $\begin{array}{c}\text { Unter } \\
\text { Bewährungshilfe } \\
\text { oder } \\
\text { Führungsaufsicht } \\
\text { stehende Probanden }\end{array}$ & $\begin{array}{c}\text { Stellen für } \\
\text { Bewährungshelfer }\end{array}$ & $\begin{array}{c}\text { Probanden } \\
\text { je Stelle }\end{array}$ \\
\hline Baden-Württemberg & 20.626 & 242,2 & 85,0 \\
Bayern & 20.295 & 270,0 & 75,2 \\
Berlin & k.A. & k.A. & k.A. \\
Brandenburg & 5.580 & 95,0 & 58,7 \\
Bremen & k.A. & k.A. & 65,8 \\
Hamburg & 4.062 & 61,9 & 70,4 \\
Hessen & 11.473 & 163,0 & 81,1 \\
Mecklenburg-Vorpommern & 4.868 & 60,0 & 62,0 \\
Niedersachsen & 15.552 & 251,0 & 62,2 \\
Nordrhein-Westfalen & 40.763 & 655,0 & 88,2 \\
Rheinland-Pfalz & 8.869 & 100,5 & 77,8 \\
Saarland & 2.411 & 31,0 & 81,1 \\
Sachsen & 7.549 & 93,1 & 78,5 \\
Sachsen-Anhalt & 6.278 & 80,0 & 71,8 \\
Schleswig-Holstein & 4.126 & 57,5 & 84,2 \\
Thüringen & 4.376 & 52,0 & $\mathbf{7 0 , 8}$ \\
\hline Insgesamt & 156.828 & $\mathbf{2 . 2 1 2 , 8}$ & \\
\hline
\end{tabular}

${ }^{*}$ Quelle: Hessisches Ministerium der Justiz, 5111/27 - 1/9 - 196/01 
Jehle, J.-M.; Sohn, W. (1994) (Hrsg.): Organisation und Kooperation der Sozialen Dienste in der Justiz. Wiesbaden, Kriminologische Zentralstelle.

Kurze, M. (1999), Soziale Arbeit und Strafjustiz. Wiesbaden: Kriminologische Zentralstelle.

\section{Materialien:}

Statistisches Bundesamt: Bewährungshilfestatistik 1998/99, Fachserie 10 / Reihe 5.
$B M I / B M J$ (2001): Erster Periodischer Sicherheitsbericht. Berlin (ins. S. 394 - 407) www.bmj.bund.de.

Justizministerium Niedersachsen: Angaben zu den Sozialen Diensten Niedersachsens www.mj.niedersachsen.de.

Peggy Schäpler ist wissenschaftliche Mitarbeiterin am Lehrstuhl für Kriminologie an der Universität Greifswald

\section{Anmerkungen}

1 Vgl. Kurze, M. (1999, S. 32ff.); Diskussionen hinsichtlich einer Änderung der Organisationsstruktur gibt es derzeit in Baden-Württemberg vor dem Hintergrund der geplanten Privatisierungen und in Niedersachsen.

2 Vgl. Dünkel, F.; Geng, B.; Kirstein, W. (1998).

$3 \mathrm{Vgl}$. BMI/BMJ (2001, S. 400).

4 Vgl. hierzu insbesondere $B M I / B M J$ (2001, S. $400 \mathrm{ff}$.).

5 Vgl. Dünkel, F.; Spieß, G. (1992, S. 121).

6 Vgl. Block, P. (1997, S. 197).

7 Vgl. Kurze, M. (1999, S. 485)

\section{Gerichtshilfe - auf halbem Wege? Gerichtshilfe als Teil der Sozialen Dienste der Justiz}

\section{Stefan Thier}

U nter dem Sammelbegriff der (ambulanten) Sozialen Dienste der Justiz werden üblicherweise die Säulen Bewährungshilfe, Führungsaufsicht und Gerichtshilfe zusammen gefasst. Mit Ausnahme der aktuellen BadenWürttembergischen Initiative zur »Privatisierung der Bewährungs- und Gerichtshilfe (dortige Pressemitteilung vom 26.11.2003: Die Regierung wird ein Pilotprojekt der Übertragung der Sozialen Dienste der Justiz (Bewährungs- und Gerichtshilfe) durchführen mit dem Ziel einer generellen Übertragung auf privat-rechtlich organisierte Vereine) gilt für diese Säulen, dass ihre Mitarbeiterinnen und Mitarbeiter der jeweiligen Landesjustizverwaltung angehören.

Sämtliche Maßnahmen der Sozialen Dienste der Justiz sind Bestandteil des justizförmigen Verfahrens und beinhalten insbesondere Kontroll- und Ermittlungsaufgaben sowie Berichtspflichten. Das sogenannte doppelte Mandat gilt für die Bewährungshilfe ebenso wie für die Gerichtshilfe, deren Aktivitäten nicht ausschließlich auf die beschuldigte oder verurteilte Klientel ausgerichtet sind, sondern den justiziellen Auftraggeber und dessen Erwartungen zu berücksichtigen und zu bedienen haben. Insofern erfolgen soziale Hilfen für die Klientel ebenso wie die justizförmige Kontrolle.

Im Sozialen Dienst der Gerichtshilfe treffen wir in den Ländern (im Gegensatz zur Bewährungshilfe) auf ein äußerst uneinheitliches Erscheinungsbild, das sich nicht nur auf die spezifischen Organisationsformen und Rahmenbedingungen beschränkt. Während die Sozialen Dienste der Justiz in den einzelnen Bundesländern zwar unterschiedlich organisiert sind (z. T.
Anbindung der Bewährungshilfe an die Landgerichte, der Gerichtshilfe an die Staatsanwaltschaften, z. T. einheitlicher Sozialer Dienst), deren Vielfalt darzustellen den Rahmen sprengen würde, so gelten für die »klassische« Bewährungshilfe doch weitgehend die gleichen gesetzlichen Rahmenbedingungen und die gleichen inhaltlichen und fachlichen Methoden, Standards und Angebote.

Die Gerichtshilfe hingegen litt und leidet unter den bundesrechtlichen Grundlagen, die zwar die Möglichkeit der Beteiligung der Gerichtshilfe vorsehen, deren Einschaltung aber im Ermessen des jeweils zuständigen Staatsanwaltes oder Richters auf der Grundlage der Strafprozessordnung liegt. Die zurückliegende Entwicklung der Gerichtshilfe hängt maßgeblich mit diesem gesetzlichen Hintergrund zusammen. Die weitere fachliche Entwicklung und Relevanz der Gerichtshilfe im Gesamtkontext von Straffälligenhilfe und Kriminalpolitik wird jedoch weniger von der Veränderung der strafprozessualen Rahmenbedingungen, als vielmehr von der strategischen Gewichtung dieses Dienstes in den einzelnen Landesjustizministerien und von der Bereitschaft und Fähigkeit der Fachkräfte abhängen, sich zu einem unverzichtbaren Dienstleistungsanbieter der Straffälligenhilfe (weiter-) zu entwickeln.

\section{Bisherige Entwicklung}

Während die Bewährungshilfe aktuell in vielen der alten Bundesländer ihr 50-jähriges Bestehen feiert, erfolgte die Einführung der Gerichtshilfe erst zum 01. Januar 1975 (Art. 294 EGStGB). Dadurch wurde einerseits anerkannt, dass ein tä- terorientiertes Strafrecht auch Ermittlungen zur Person des Beschuldigten/Verurteilten umfassen soll, mit denen die Gerichtshilfe beauftragt werden kann. Allerdings ging der Gesetzgeber nicht so weit wie bei der Verankerung der Jugendgerichtshilfe. Analog zur JGH wurde die Gerichtshilfe zwar ebenfalls institutionell verankert, doch erfolgten keine konkreten Aussagen zu den Aufgaben der Gerichtshilfe und zu ihrer strafprozessualen Stellung.

Die Strafprozessordnung erwähnt die Gerichtshilfe in den $\S \S 160$ Abs. 3 und 463 d. In beiden Fällen ist sowohl im Ermittlungs- wie auch im Vollstreckungsverfahren der Einsatz der Gerichtshilfe vorgesehen, doch bleibt deren Einsatz aufgrund der »Kann-Bestimmungen « vage und unverbindlich. Sowohl die Staatsanwaltschaft (§ 160 Abs.3) wie auch das Gericht oder die Strafvollstreckungsbehörde ( $\$ 463$ d) können sich der Gerichtshilfe »bedienen «. Diese doch recht groben Umschreibungen haben den Landesgesetzgebern seit 1975 einen erheblichen Gestaltungsspielraum zum Einsatz der Gerichtshilfe ermöglicht. Diese Möglichkeit, den Einsatz eines Sozialen Dienstes länderspezifisch und flexibel zu gestalten, hat dem Berufsbild der Gerichtshelferin und des Gerichtshelfers eher geschadet. Im Gegensatz zur Jugendgerichtshilfe ist es der Gerichtshilfe in den nunmehr zurückliegenden knapp 30 Jahren nicht gelungen, im Rahmen des Ermittlungs- und Hauptverfahrens die Bedeutung und das Profil der Jugendgerichtshilfe zu erlangen.

Die Gerichtshilfe heute zu skizzieren fällt weniger aufgrund der in den Ländern unterschiedlichen Organisationsformen schwer, vielmehr haben landesgesetzliche Regelungen (Tilgungsver- 